\title{
DIGITAL MINING:
}

\section{COMO NEXA RESOURCES ESTÁ CONSTRUINDO A}

Nexa Resource Team ${ }^{1}$

A digitalização não é mais uma novidade. De fato, é um processo que segue como evolução natural da aplicação do conhecimento que tem sido construído há décadas. Contudo, a aceleração causada pela Covid é sem precedentes, pois mudou a forma com que as pessoas se relacionam. E é essa alteração no tecido social que é o aspecto mais relevante quando se discute esse tema. A digitalização muda a forma de lidar com dados, processos e, mais importante, uns com os outros. A democratização do acesso a ferramentas, a onipresença virtual e a simplicidade de acesso a informações promovem a geração de conexões não óbvias: a fagulha fundamental da inovação. Gerir a mudança é o aspecto que pode garantir o sucesso das organizações. Cenários incertos ou alternativas de baixa probabilidade são parte do dia a dia e adaptar o comportamento reorganizando-se à medida que a realidade apresentada se altera é fundamental. Neste texto é discutido como o conjunto de pessoas com um objetivo comum - a Nexa - enxerga a digitalização como caminho para desenvolver a sua estratégia ESG, os aspectos de mudança cultural promovidos e casos de aplicação.

A quarta revolução industrial, ou Indústria 4.0, não é um dos temas mais recentes em discussão entre as empresas da indústria de base. Entretanto, os campos de aplicação e usabilidade das tecnologias trazidas junto com esse novo ciclo crescem a cada dia mais. Ao mesmo tempo, o aumento da relevância de temas ligados a aspectos ambientais, sociais e de governança (ESG) faz com que a atenção da organização se expanda para além das suas atividades principais de produção ou corporativas.

A busca de tecnologias para digitalização tem o objetivo de trazer às pessoas mais tempo para o desempenho de atividades estratégicas, com a redução do tempo empreendido em atividades operacionais. Enquanto isso, a cultura de inovação tem como base as pessoas que são responsáveis pela identificação dos processos digitalizáveis e a implantação da transformação.

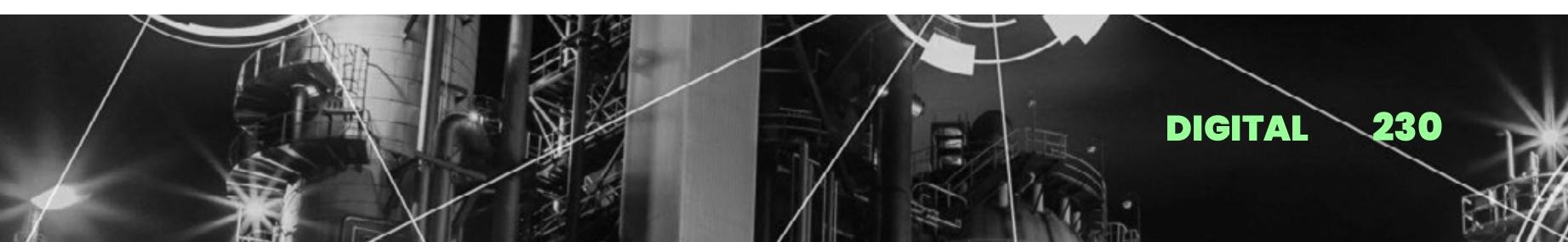





\section{A platcforma, ctualmente, permite a rápida enálise 20 de}

deles geológlices, geoqúfmicos, geofísices e outros. Depois de uma preparaçăo da base de dades, feita a partir de algoritmos especifices desenvolvidos parra essa funģão, é possível avalíar árecrs de até $25.000 \mathrm{~km}^{2}$ em poucess hords. com a implementação desta ferromenta, pode-se cumentar a produtividade dos profissionalis envolvidos nas atividades de exploraçấ minerel, inerementer a assertividade das regifes a serem avaliadas, gerendo um menor impacto a๑

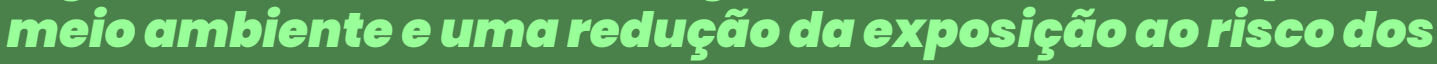
colcboradores Nexa.

\section{DIGITALIZAÇÃO DE CHECK-LISTS}

Antes de iniciar as atividades em campo, os membros do time de Exploração Mineral tinham de realizar um check-list das condições de uso de seus veículos. O check-list, realizado em papel para avaliação preliminar, era seguido de digitalização manual da informação. A ausência de uma avaliação em tempo real dos dados e a necessidade de transferir do papel para uma base digital não garantiam a agilidade necessária para as avaliações de segurança. Com isso, foi lançado ao ecossistema de inovação um desafio para identificação de uma ferramenta digital que tivesse todas as funcionalidades para o uso do formulário e que funcionasse sem a disponibilidade constante de sinal de dados, haja vista a necessidade de uso em regiões remotas. Após a identificação do parceiro estratégico para construção dessa solução, foi realizada a PoC com um pequeno grupo de usuários para adaptação da ferramenta e construção de um formulário capaz de atender as necessidades das equipes. Com a validação e aprovação da ferramenta em pequena escala em um projeto em campo, seu uso foi expandido para os demais projetos da Exploração Mineral. Vendo o potencial que a ferramenta oferecia, foi replicada para os demais check-lists existentes na área. Em uma primeira onda de implantação, mais de 150 check-lists estão sendo colocados em produção para Brasil, Peru e Namíbia. 0 diferencial que podemos trazer com o uso dessa ferramenta, que funciona de maneira remota tanto para Android quanto para IOS, é a gestão dos dados em campo de maneira estruturada, onde é possivel avaliar as não conformidades detectadas durante a realização do preenchimento do check-list, tendo em tempo real as notificações diretamente para os gestores de cada projeto e podendo acompanhar por meio de um portal o andamento de todos os processos que estão sendo realizados em campo.

Após o início da pandemia de COVID-19, foi visto dentro das funcionalidades desse aplicativo a possibilidade de autoavaliação da saúde dos colaboradores da empresa, garantindo um acompanhamento próximo e ágil da equipe de saúde e segurança e suporte médico quando necessário. Hoje, a ferramenta conta com mais de 10 áreas de toda a Nexa em ambiente de teste ou uso definitivo da ferramenta. 


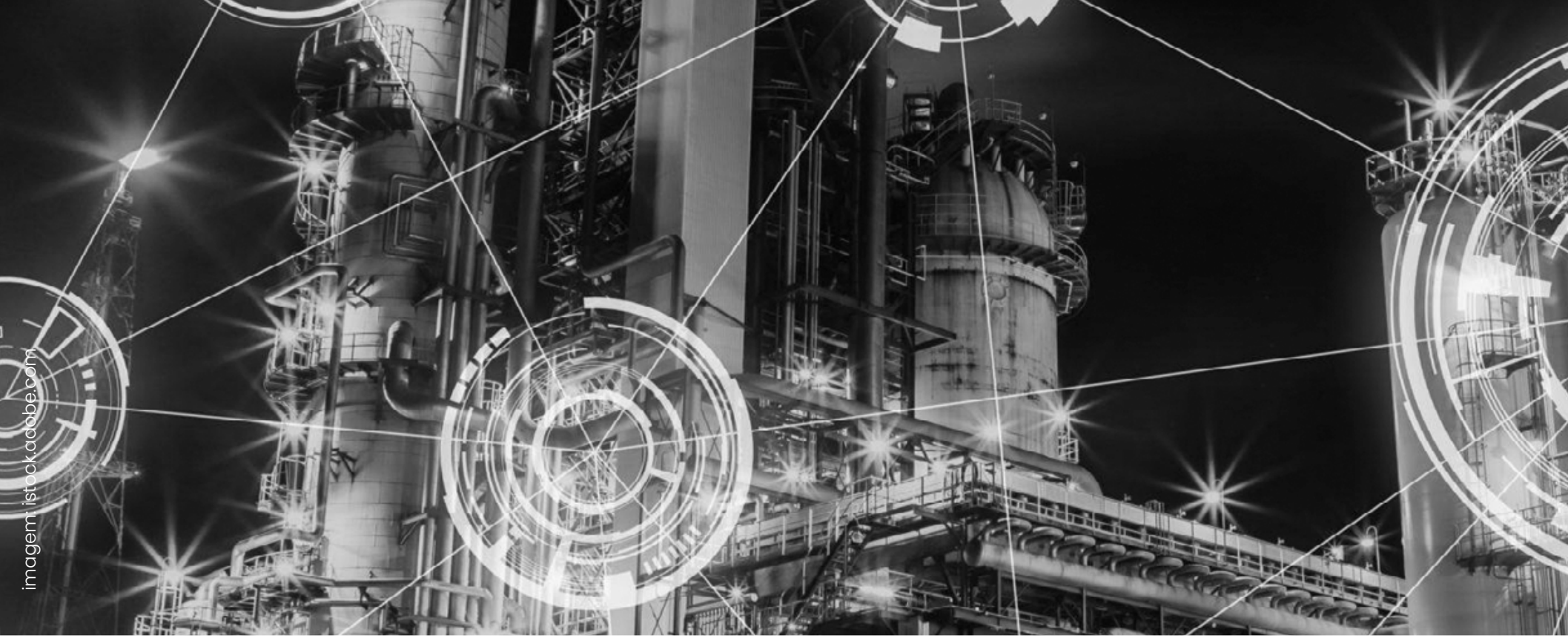

\section{DIGITALIZAÇÃO de DADOS GEOTÉCNICOS}

Avançando na cadeia produtiva do zinco, entramos nas atividades de mineração. Na mineração subterrânea, a avaliação estrutural das frentes de lavra é uma atividade de grande importância e demanda uma alta quantidade de dados precisos para que se possa realizar o planejamento dos estopes. Esse levantamento, usualmente feito de forma manual, demanda muito tempo dos colaboradores em atividades operacionais, além de estar sujeito à incerteza de medição.

Diante desse cenário, foi lançado ao mercado, por meio da Plataforma Mining Lab, um desafio para busca de soluções inovadoras para essa atividade. A parceira estratégica selecionada para desenvolvimento da solução propôs a construção de um equipamento robusto, prático e eficiente. Com esse equipamento é possível realizar a medição da inclinação e orientação das superfícies e trincas em poucos segundos e à distância. A obtenção das mesmas informações manualmente é sujeita a erros de leitura instrumental, à propagação de erro pelas diversas medidas e não pode ser feita à distância, sendo uma grande vantagem o uso do equipamento.

\section{Com dados precises e malis tempo disponível, os celalboradores podem desenvolver planejelmentos de construģấ de gelerics aind a mais efieientes.}

Inicialmente, esse projeto foi desenvolvido na operação de Morro Agudo em Paracatu (Minas Gerais) e, hoje, está sendo testado na operação de Cerro Lindo, no Peru. A partir do uso desse equipamento pode-se realizar uma coleta de dados com até três vezes mais pontos em cerca de um oitavo do tempo necessário para uma medição manual e em até vinte metros de distância da frente de lavra, tendo em seguida a projeção estereográfica gerada automaticamente. Assim, é possivel garantir dados mais precisos para as atividades de desmonte e reforço estrutural, aproveitando ainda mais os recursos minerais disponíveis, garantindo alicerces ainda mais precisos para um ambiente de trabalho cada vez mais seguro. 


\section{DESMONTE COM DETONAÇÃO REMOTA}

Após a avaliação estrutural da frente de lavra, é planejado e executado o seu desmonte, permitindo, assim, o manuseio do minério. Para a execução da atividade de desmonte, é necessário um bom dimensionamento da quantidade de explosivo a ser utilizado e uma avaliação estrutural robusta, além de garantir que não existam pessoas no interior da mina, conferindo a sua saída e bloqueando a entrada para depois o blaster retornar e acionar/iniciar a detonação. Dessa forma, com o objetivo de trazer um rápido retorno para o processo de desmonte de pilares e torná-lo mais eficiente, como também mais seguro, iniciou-se o projeto de desmonte com detonação remota através do CEBS, que é um sistema de detonação eletrônico centralizado que permite ao colaborador iniciar o desmonte subterrâneo a partir de um local remoto ou até mesmo da superfície utilizando um computador/tablet/caixa de detonação.

Por ser uma tecnologia pioneira no Brasil e ainda pouco utilizada no mundo, a Nexa enfrentou desafios como garantir uma adequada disponibilidade do sistema de comunicação da mina subterrânea. O projeto de retomada de pilares, por ser sensível e possuir risco adicional, necessitou incluir sistema de monitoramento de microssismos e LHDs (load, haul and dump) operados por controle remoto. O desenvolvimento do projeto levou cerca de um ano e foi possível obter resultados em linha com os objetivos esperados, como detonação simultânea de diversas frentes, maior segurança para operadores e blasters e redução do tempo total para atividade de detonação. Inicialmente, foram realizadas provas de comunicação na superfície por meio de validação de alguns requisitos para o sucesso do projeto, como, por exemplo, determinação das frequências, posicionamento da antena, do modem repetidor e do head end.

Com o sucesso de todas as etapas de teste e a possibilidade de ganhos em diversas áreas que acercam o desmonte, a implantação se mostrou como um caminho natural para o projeto.

\section{- principal geinho eneontra-se no cumento de segureinģa} dessa atividade parra todos os coleboradores envolvidos, cssim eomo na redução no tempo dos troecs de turno, reduzindo o tempo de eielo, devide do foto de terrefos de rotina porra d execução do desmonte levarem menos tempocom essa nova teenologia.
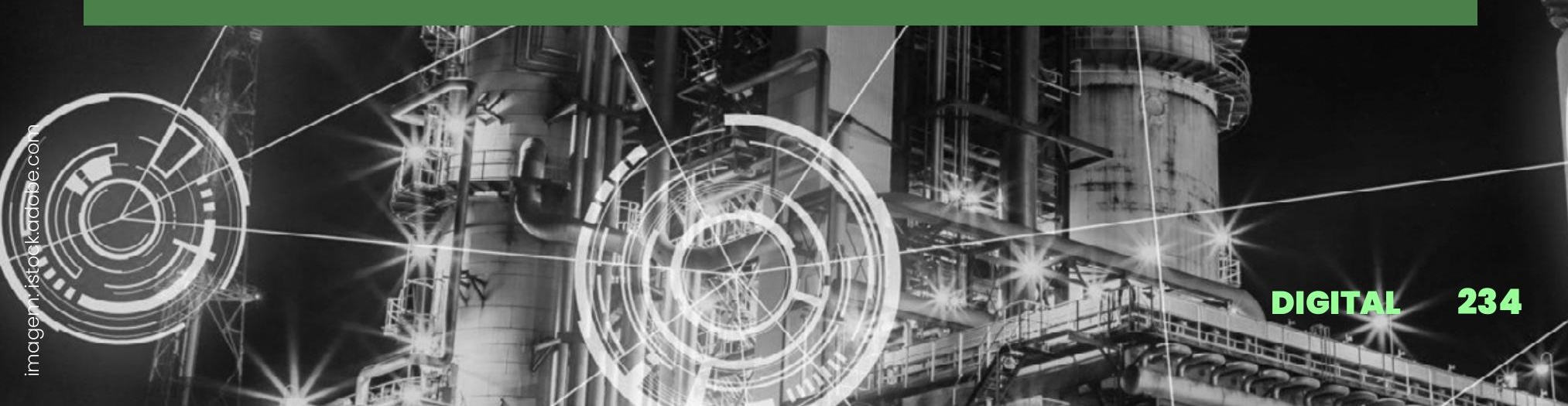
O sucesso dessa iniciativa em Morro Agudo permitiu a sua expansão em outras operações da companhia, como a de Vazante e Cerro Lindo.

\section{USO DE DRONE NA MINERAÇÃo}

Visando diminuir o tempo necessário para realizar levantamentos topográficos pela equipe de geologia em cavas, foram desenvolvidos testes com o uso de drones na operação de Cerro Lindo, no Peru. Como primeiro passo do projeto, foram avaliadas soluções já existentes no mercado, buscando-se drones autônomos que possuíam equipamentos de fotometria. Após a seleção do equipamento mais pertinente, foram realizados testes de campo. Sem grandes obstáculos e com uma hora de trabalho com o drone, já era possível obter um cálculo volumétrico que levaria cerca de um dia para ser feito com outros métodos, como com o deslocamento pela área com o uso de caminhonetes a diesel.

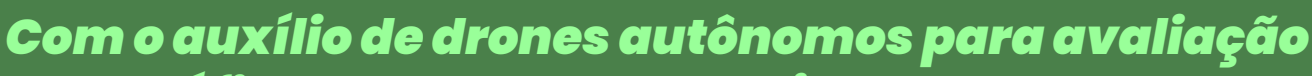
topográfied, pode-se gerar mais dedes peIra suporte a०

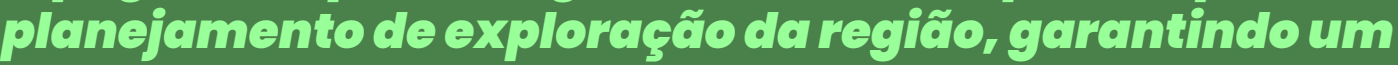
aumento de assertividade e produtividade das equipes, além de evitar o use de combustíleis come diesel e querosene de aviação, reduzindo a emissão de GE三 (gases de efeilı estuf́a).

\section{IMPRESSORA 3D}

Outro projeto desenvolvido dentro das plantas de mineração da companhia é o uso de impressoras 3D. Esse projeto, desenvolvido em Vazante, surgiu com a finalidade de viabilizar a fabricação de peças plásticas das mais diversas áreas, com o objetivo de redução de custos e leadtime com a aquisição no mercado. Mapeando soluções já existentes no mercado, a Nexa realizou a seleção de sua parceira estratégica para PoC (prove of concept), dando preferência a parceiros com experiência prévia em modelamento 3D de peças já existentes. Após a construção do modelo 3D da peça desejada e dos testes mecânicos do modelo fabricado, foi validada sua produção dentro da própria planta sem detrimento de sua qualidade.

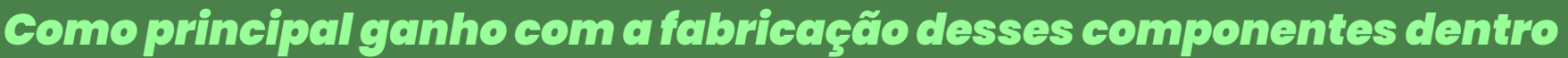
da própria mina, é possível destacer a recuperaçčo dos componentes eletrônicos do eontrole remoto e eusto evitddo de 5 mil rectis por

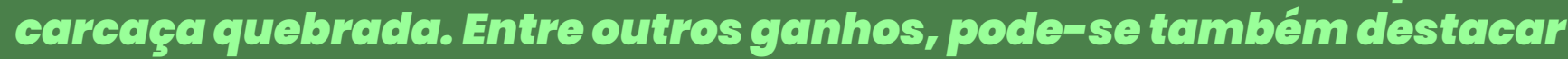
a possibilidade de reduzir eareaças em estoque, visto menor eusto de estocegem, redução do volume de resíduos eletrônicos gerados e possibilidade de produģăo de outras peģes com a impressorc. 


\section{REDUÇÃO DO CUSTO DE ENERGIA EM CAJAMARQUILLA}

Após as etapas de extração e concentração mineral nas plantas de mineração, o concentrado de zinco é encaminhado para a planta de metalurgia, onde por processos piro e hidro metalúrgicos é produzido o zinco metálico. A principal rota de produção de zinco no mundo é a eletrorrecuperação, gerando consumo de energia elétrica e chegando a ser $80 \%$ do consumo total da planta industrial.

O custo de energia elétrica varia ao longo do dia, sendo maior nos momentos de mais alta demanda. A equipe de processos de Cajamarquilla, com o objetivo de reduzir os custos de operação, realizava manualmente um estudo do cenário de consumo elétrico da operação e, com essa informação, planejava a operação da planta para trabalhar em períodos de menor custo de energia. Com o objetivo de otimizar essa atividade, foi desenvolvido um algoritmo computacional para avaliação dos dados de consumo energético peruano integrado aos sistemas de dados da operação. Esse sistema faz a leitura do consumo da planta e fornece apoio ao planejamento da operação, promovendo a redução de custos.

com॰ principel genho dessc etiped do projeto houve o eusto evitado de multas consequentes de consumo acima do plenejado em horćtrios de pico em USD 1,67 milhã॰ c० ch०e reduçấ de 0,4 FTE (full time employee).

Como segunda etapa desse projeto, foi desenvolvido um modelo de machine learning para predição da curva de consumo do sistema energético peruano e uma prescrição de atuação para a área estratégica de energia da Nexa, conciliando as condições de contorno para atingir um menor custo de operação.

\section{TENDÊNCIAS TECNOLÓGICAS}

As atividades de digitalização vão muito além das nossas operações de mineração e metalurgia. A produção de conhecimento com a publicações de artigos científicos é cada vez mais dinâmica, com novos conhecimentos sendo expostos a todo momento. Da mesma forma, quando uma empresa busca garantir o direito de exploração de um processo ou produto por meio de uma patente, pode-se ver os próximos passos da transformação do mercado. O volume de informações novas disponibilizadas é cada vez maior, o que faz com que a análise manual tome tempo demais para ser conduzida.

Neste cenário, e com o objetivo de manter a Nexa atualizada do estado da arte do conhecimento, foi desenvolvida uma ferramenta baseada em aprendizado de máquina e inteligência artificial. 
Essa ferramenta consegue ler e priorizar a informação de diversas fontes, reduzindo em ordens de grandeza o material que nossos especialistas devem avaliar. A criação desse analisador de patentes e artigos foi feita pela Nexa, em conjunto com desenvolvedores de software, identificados na segunda edição do Mining Lab Challenge ${ }^{2}$, em 2018.

Com a dplicação dessa teenologid, pode-se identifiecr de forma rópída quais sc̃o os pafíses, centros de pesquisa e empresas que maís têm produzide conhecimento, além de priorizar es temas mais eitcrdes.

Com isso, vemos onde tópicos do nosso interesse são mais estudados e podemos nos certificar que o nosso portfólio também contém pesquisas e aplicações das mais recentes inovações. Anualmente, apresentamos para a direção da empresa um resumo daquilo que foi visto e o que se deseja trazer para a empresa de forma a auxiliar o processo de tomada de decisão sobre investimentos. Essa ferramenta nos ajuda na construção da nossa visão da mineração do futuro.

\section{GESTORA VIRTUAL DO CONHECIMENTO}

Com o aumento da quantidade de projetos e parcerias desenvolvidas com equipes externas à Nexa, a demanda do time jurídico pelo envio de modelos e avaliação de contratos de transferência tecnológica, proteção de propriedade intelectual ou dados de procurações, entre outros, também aumentou.

Assim, foi lançado ao mercado um desafio para busca de soluções para automatizar e gerir o conhecimento jurídico da empresa, de forma a permitir que os especialistas legais possam investir mais tempo em atividades menos repetitivas.

\section{A partir de seleção do pareeiro teenológie de seluçčo, iniciou-se o processe de desenvolvimento de Nind - a gestord virtual do conheeimento de Nexa, que responde dúvidas e envia doeumentos padrão perra validação e djustes inicicis por parte do fornecedor.}

Após a validação da ferramenta com o conteúdo da área jurídica, suas aplicações foram levadas para outras áreas, como Desenvolvimento Humano e Organizacional e Auditoria. 


\section{PRÓXIMOS PASSOS}

A transformação digital da indústria da mineração é uma tendência irreversível. As principais empresas do setor têm se debruçado em desafios sobre como aumentar o tempo que seus colaboradores gastam em análise de dados e diminuir o tempo com interações operacionais. Diminuição de riscos e melhoria do desempenho operacional também se tornaram o foco do investimento dos principais players desse mercado. Não obstante, temas como ESG também fazem parte da agenda das mineradoras, atendendo também às demandas de suas principais partes interessadas, incluindo, mas não se limitando, acionistas, comunidades, órgãos reguladores, parceiros da cadeia de suprimentos e clientes. Essas demandas se materializam em projetos como: monitoramento contínuo de taludes com inteligência artificial, monitoramento online da localização e saúde de colaboradores, de forma a ser possível monitorar condições de operação e trabalho e simulação online de processos, para redução no consumo de matériasprimas e reagentes.

O caminho para construção da mineração do futuro é longo e desafiador. A mentalidade de digitalização de processos e a estratégia de seleção/priorização dos mesmos, alinhados aos objetivos da corporação, podem trazer resultados rápidos e consistentes. O uso de provas de conceito pode ajudar no modelamento das etapas necessárias para scale up, aumentando os impactos positivos do portfólio de projetos dentro da organização.

\section{A transformaģão digitid de uma orgennização de porte} da Nexa não está vineulada somente às tendêneics tecnológicas e à estratégị da empresa, mas a questão eultural passa a ser erueicl para o sucesse des iniciativas. Do ponto de vista de gestão de pessods

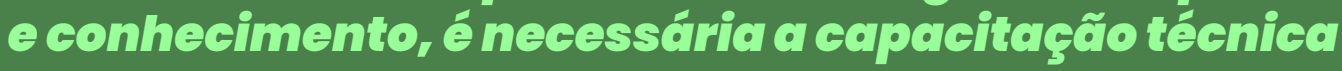
e eultural de todo o grupo parra eonstrugấo de uma eultura digitul peira manutenģăo dos resultades de projetos.
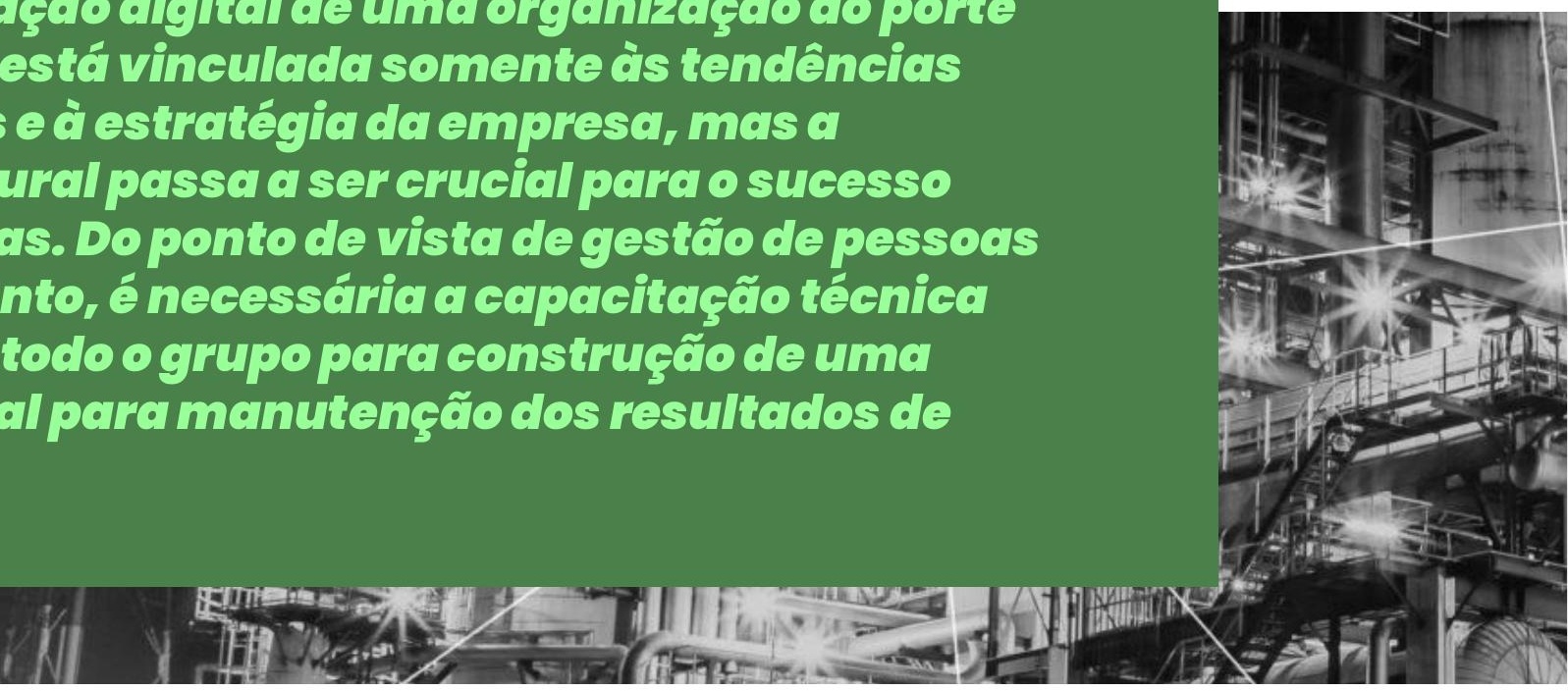

Por fim, momentos como este de pandemia fazem com que sejam revistas prioridades, mas também são incentivos à identificação de novas oportunidades de digitalização, evidenciando a necessidade de experiência e pragmatismo para gestão de portfólio de projetos. 


\section{Nexa Resource Tecm}

Allan Fruchting; Ana Paula Mendonça Bouchardet; Bruno Baptistella; Caio Moreira van Deursen; Daniel Henrique Soares; Douglas dos Anjos Silva; Fabio Pereira De Miranda; Felipe Goes; Fidel Fidelix; Flávio Padula; Francisco Carlos Ferreira Ila; Frederik Stadmann, Gilberto Hashimoto; Glauco Malagoni; Henrique Souza Fascini; Jimmy Frank Leon; João Henrique Boniatti; Joyce Teixeira Gardel Barrancos; Luiz Fernando Marcondes; Luiz Guilherme Stipp Correia; Marco Henrique Carrete; Mariana Naomy; Matheus Pelichek Bueno; Mauro Teixeira Soares; Monica Bezerra; Moisés Magnus Bizinoto Rafael Murca; Rafael Nonato; Rafael Skupek; Richard Ordoñez; Rodrigo Alberto Moreira Gomes; Rodrigo Rossi Viana; Thiago José de Medeiros Silva; Victor Coqueiro Ferreira; Vinicius Xavier.
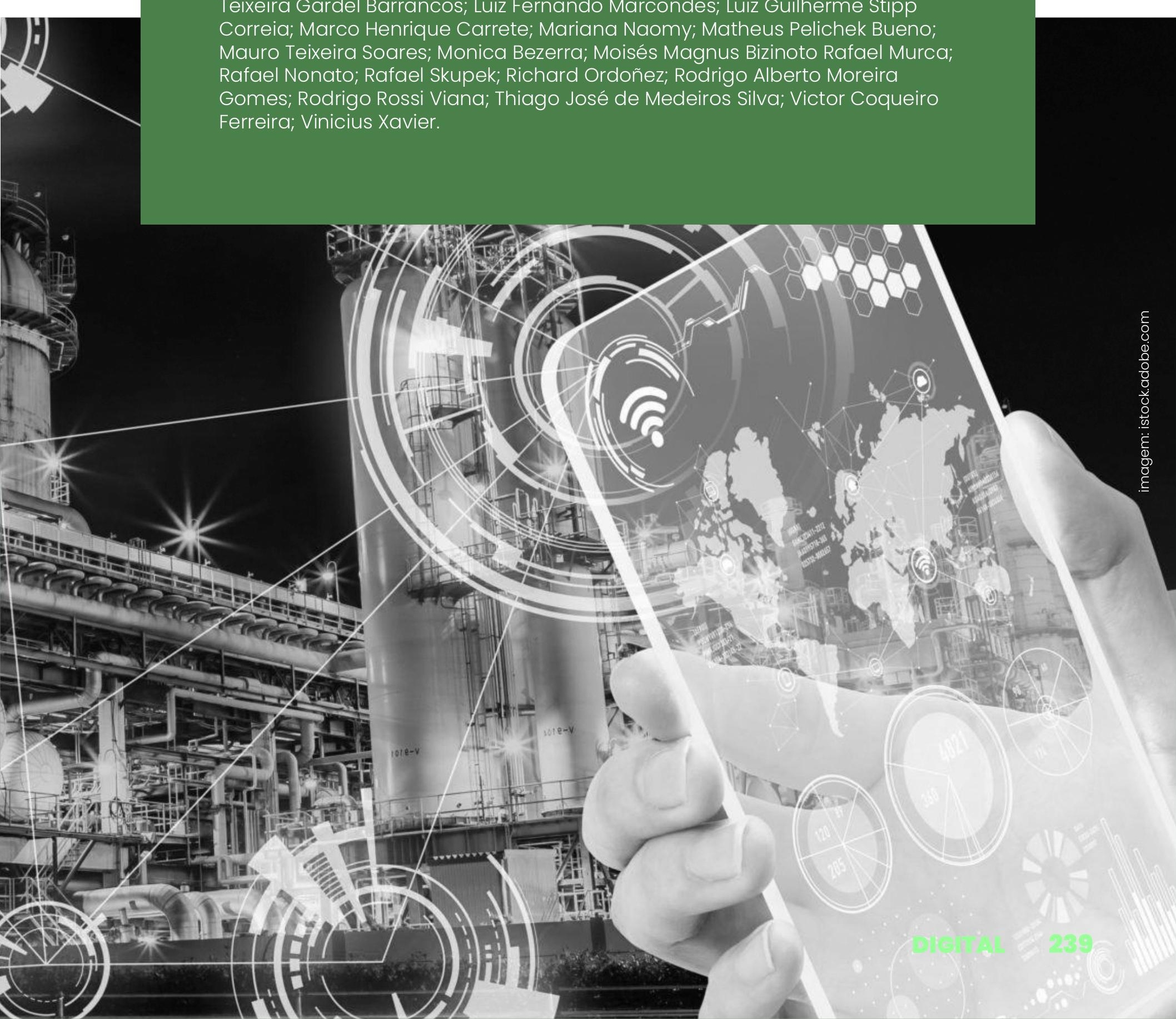


\section{NOTASEREFERÊNGIAS}

1 O presente texto é de responsabilidade da equipe da Nexa responsável pela implementação da estratégia de transformação digital da empresa.

2 O programa Mining Lab Challenge foi criado pela Nexa para atrair e selecionar iniciativas de empreendedores que desejam desenvolver projetos de inovação tecnológica para a indústria de mineração e metalurgia. Ver https://www. mininglab.com.br/ 